Research Article

\title{
Global Stability of Switched HIV/AIDS Models with Drug Treatment Involving Caputo-Fractional Derivatives
}

\author{
Xiying Wang $(D$, Wenfeng Wang, and Yuanxiao Li \\ College of Science, Henan University of Technology, Zhengzhou 450001, China \\ Correspondence should be addressed to Xiying Wang; wangxiying668@163.com
}

Received 12 November 2020; Revised 14 January 2021; Accepted 29 January 2021; Published 18 February 2021

Academic Editor: Ya Jia

Copyright (c) 2021 Xiying Wang et al. This is an open access article distributed under the Creative Commons Attribution License, which permits unrestricted use, distribution, and reproduction in any medium, provided the original work is properly cited.

In this paper, we formulate and investigate new switched HIV/AIDS models with drug treatment involving Caputo-fractional derivatives. Initially, due to the fractional derivative order related to the memory and hereditary effects and supposing that the model coefficients are time-varying parameters, we develop a Caputo-fractional order HIV/AIDS models with switching parameters and study their dynamics utilizing Lyapunov-Razumikhin technique. Furthermore, the results show that the fractional derivative $\alpha(0<\alpha<1)$ and the switching parameters are related to the critical threshold value $(\widehat{R}$ or $\bar{R})$ which ensures disease eradication under the condition of $\widehat{R}<1$ or $\bar{R}<1$. Then, a treatment compartment is introduced into the above model from the asymptomatic infected individuals until the full blown AIDS individuals. Novel sufficient conditions on the threshold value are derived to verify that the disease is eventually cleared as the critical threshold parameter is below unity. Finally, some simulations are employed to support the main results and one future research direction is presented.

\section{Introduction}

HIV/AIDS has been one of the most deadly diseases around the world since the first patients were documented in 1981. It has seriously affected human health and even life. According to clinical trials, the virus will go through life once infected with HIV. In the absence of drug treatment, the average life span of patients from HIV infection to AIDS is within 5-10 years [1]. With effective drug therapies, treated individuals can prolong their survival and improve their life quality but do not cure for HIV or AIDS. Thus, it is necessary to propose effective methods for prevention and control of AIDS.

In recent years, many epidemic issues have been studied by modelling mathematical models to reveal transmission of the disease or predict tendencies of the disease. In particular, Anderson et al. [2] investigated the dynamics of the initial HIV models using ordinary differential equations. The models have been improved by adding into various factors on the disease [3-5]. Okosun et al. [6] developed HIV/AIDS with treatment and screening of unaware infectives and investigated optimal control of the treatment. Pitchaimani and Monica [7] incorporated three time delays into a HIV-1 infection model and gave the existence of Hopf bifurcation of the model. Silva and Torres [8] introduced the fractional order into an HIV/AIDS model and surveyed local and uniform stability of its disease-free equilibrium. Huo et al. [9] added anti-HIV preventive vaccines into a fractional order HIV model and showed that the model has rich phenomenon of backward bifurcation with different dosages of vaccines and fractional derivative orders. In their models, they show that fractional order models are related to memory, history, or nonlocal property, which seems to better display complex behavior of real-world phenomena. Nowadays, fractional order models have been wide applied in many fields such as applied mathematics, engineering, economics, biology, and medicine [10-12]. Modelling the dynamical behavior of the epidemic diseases by fractional derivative models has more effective than interorder modelling [13-15]. For instance, Ullah et al. [16] studied the dynamics of fractional order derivative tuberculosis model and proved the existence and uniqueness of equilibria. Kheiri and Jafari [17] proposed a multipatch fractional order derivative epidemic model and derived relationship between the value of objective functional and the fractional derivative 
order. Khan and Atangana [18] formulated novel corona virus models with fractional derivative and studied the relationship between the fractional order parameters and the infected compartments. Naik et al. [19] modelled a fractional order HIV-1 model and verified the existence of equilibria and their asymptotical stability.

Seasonal changes in a fluctuating environment often have an effect on the transmission of an infectious disease, which naturally causes that the biological and environment parameters are varying in time. For instance, the spread of dengue disease (transmitted by Aedes aegypti) is fast in summer, and the spread is low in the winter. It has been shown that the transmission speed of childhood infections is the highest at the start of the school year and then declines gradually $[20,21]$. The research works on the infection disease models affected due to seasonality (such as changes in host immunity or host behavior) have been carried out [22-24]. In most of the epidemic models, the coefficients are traditionally assumed to be constant or smoothly varying functions. However, in some cases (such as vacation for school children), the models parameters are assumed to change abruptly in time [25-28]. This type of parameter is called switching parameter, and the model is called the switched system. Since this type of epidemic model involves multiple subsystems and subject investigated is relatively complex, research work is relatively few [29-31]. Thus, it is extremely significant to incorporate switching into epidemic models and study their dynamics.

This paper mainly develops and investigates switched HIV/AIDS models with drug treatment involving Caputofractional derivatives. Specifically, the models' parameters under the influence of seasonality are supposed to be varying in time and change their function forms, and the derivative of the model is assumed to be fractional order with its memory property, which leads to a novel HIV/AIDS model. New global stability conditions of the disease-free equilibrium are derived to examine the dynamics of the disease based on Lyapunov-Razumikhin technique. Moreover, treatment strategies are proposed and evaluated to show that the drug therapy has important influence on the stability of the model.

This paper is organized as follows. The classic HIV/AIDS model is extended as a fractional switched HIV/AIDS model, and threshold conditions are obtained to illustrate that the disease-free equilibrium is globally asymptotically stable in Section 2. In Section 3, treatment strategies are applied into the above model, and sufficient conditions on the disease eradication are derived. Section 4 gives numerical examples to verify the proposed results. Conclusions are presented in Section 5 .

\section{Model Development}

Noting that memory and hereditary properties of fractional order derivatives can make complex behavioral of epidemic models, we will extend the integer order HIV/AIDS models by introducing a fractional order HIV/AIDS model with switching parameters. Assume that the total individuals $N(t)$ consist of the susceptible individuals $S(t)$, infected individuals $I_{1}(t)$ with asymptomatic, infected individuals $I_{2}(t)$ with symptomatic, and individuals diagnosed with AIDS $A(t)$, that is, $N(t)=S(t)+I_{1}(t)+I_{2}(t)$. On the contrary, the variety of seasons can made biological parameters change abruptly in time. It is assumed the model coefficients are as switching parameters. Assume these parameters are controlled by a piecewise continuous (from the left) switching signal $\sigma(t)$, in which $\sigma(t)$ is $\left(t_{k-1}, t_{k}\right] \longrightarrow\{1,2, \ldots, m\}, k=1,2, \ldots$, and $\mathscr{I}$ is the set of all switching rules. Assume that $p_{\sigma}$ is a switched transmission rate between susceptible individuals and individuals with asymptomatic, $q_{\sigma}$ is a switched transmission rate between the susceptible individuals and individuals with symptomatic, $\eta_{\sigma}$ is a switched transmission rate between the susceptible individuals and individuals with individuals diagnosed with AIDS, $e_{\sigma} r_{\sigma}$ is a switched transmission rate from individuals with asymptomatic moving to individuals with symptomatic, (1$\left.e_{\sigma}\right) r_{\sigma}$ is a switched transmission rate from individuals with asymptomatic moving to individuals diagnosed with AIDS, $\varphi_{\sigma} r_{\sigma}$ is a switched transmission rate from individuals with symptomatic moving to individuals diagnosed with AIDS, and $a_{\sigma}, b_{\sigma}$, and $c_{\sigma}$ are mortality from diseases of individuals with asymptomatic, individuals with symptomatic, and individuals diagnosed with AIDS, respectively. $\lambda$ denotes a recruitment rate of susceptible individuals. $\mu$ represents natural mortality rate of four individuals classes. Thus, the modified models is presented by

$$
\left\{\begin{array}{l}
{ }_{t_{0}}^{c} D_{t}^{\alpha} S(t)=\lambda-p_{\sigma} S(t) I_{1}(t)-q_{\sigma} S(t) I_{2}(t)-\eta_{\sigma} S(t) A(t)-\mu S(t), \\
{ }_{t_{0}}^{c} D_{t}^{\alpha} I_{1}(t)=p_{\sigma} S(t) I_{1}(t)+q_{\sigma} S(t) I_{2}(t)+\eta_{\sigma} S(t) A(t)-\left(r_{\sigma}+a_{\sigma}+\mu\right) I_{1}(t), \\
{ }_{t_{0}}^{c} D_{t}^{\alpha} I_{2}(t)=e_{\sigma} r_{\sigma} I_{1}(t)-\left(\phi_{\sigma}+b_{\sigma}+\mu\right) I_{2}(t), \\
{ }_{t_{0}}^{c} D_{t}^{\alpha} A(t)=\left(1-e_{\sigma}\right) r_{\sigma} I_{1}(t)+\phi_{\sigma} I_{2}(t)-\left(c_{\sigma}+\mu\right) A(t),
\end{array}\right.
$$

where $0<\alpha<1$. Here, the initial conditions for system (1) are $S\left(t_{0}\right)>0, I_{1}\left(t_{0}\right) \geq 0, I_{2}\left(t_{0}\right) \geq 0$, and $A\left(t_{0}\right) \geq 0$. All the parameters are assumed to be positive. For any values of the parameters of system (1), there exists a disease-free equilibrium $Q_{0}=(\bar{S}, 0,0,0)$, in which $\bar{S}=\lambda / \mu$. In the following, we first give a positive invariant set $\Omega=\left\{\left\{S(t), I_{1}(t), I_{2}(t)\right.\right.$, $A(t)\}: S(t) \geq 0, I_{1}(t) \geq 0, I_{2}(t) \geq 0, A(t) \geq 0, S(t)+\quad I_{1} \quad(t)+$ $\left.I_{2}(t)+A(t) \leq(\lambda / \mu)\right\}$. In this regard, many works have been done in the literatures $[32,33]$. And, then, we investigate the global asymptotical stability of $Q_{0}$ for system (1). Before 
giving the main results, we introduce the following definitions and lemmas of Riemann-Liouville and Caputo fractional derivative.

Definition 1 (see [34]). A Gamma function of $\alpha>0$ is defined by

$$
\Gamma(\alpha)=\int_{0}^{\infty} x^{\alpha-1} e^{-x} \mathrm{~d} x
$$

Definition 2 (see [34]). The Riemann-Liouville fractional integral of order $0<\alpha<1$ of a function $f: R^{+} \longrightarrow R$ is defined by

$$
I^{\alpha} f(t)=\frac{1}{\Gamma(\alpha)} \int_{t_{0}}^{t}(t-\tau)^{\alpha-1} f(\tau) \mathrm{d} \tau,
$$

where $\Gamma(\cdot)$ is the Gamma function.

Definition 3 (see [34]). The Caputo derivative with fractional order $0<\alpha<1$ of a function $f: R^{+} \longrightarrow R$ is defined by

$$
{ }_{t_{0}}^{c} D_{t}^{\alpha} f(t)=\frac{1}{\Gamma(1-\alpha)} \int_{t_{0}}^{t} \frac{f^{\prime}(\tau)}{(t-\tau)^{\alpha}} \mathrm{d} \tau, \quad t_{0} \geq 0 .
$$

Definition 4 (see [34]). The constant $x^{*}$ is an equilibrium point of the system ${ }_{t_{0}}^{c} D_{t}^{\alpha} f(t)=f(t), x\left(t_{0}\right) \geq 0$, and $0<\alpha<1$, if and only if $f\left(t, x^{*}\right)=0$.
Lemma 1 (see [34]). Let $f, g: R^{+} \longrightarrow R$ be such that ${ }_{t_{0}}^{c} D_{t}^{\alpha} f(t)$ and ${ }_{t_{0}}^{c} D_{t}^{\alpha} g(t)$ exist almost everywhere and let $c_{1}, c_{2} \in R$. Then, ${ }_{t_{0}}^{c} D_{t}^{\alpha}\left(c_{1} f(t)+c_{2} g(t)\right)$ exists almost everywhere with

$$
{ }_{t_{0}}^{c} D_{t}^{\alpha}\left(c_{1} f(t)+c_{2} g(t)\right)=c_{1 t_{0}}^{c} D_{t}^{\alpha} f(t)+c_{2 t_{0}}^{c} D_{t}^{\alpha} g(t) .
$$

Lemma 2 (see [34]). Let $x(t)$ and $y(t)$ be continuous realvalued functions and nonnegative in $t \in\left[\pi_{1}, \pi_{2}\right]$. If $h$ is a nonnegative constant and $x(t)$ satisfies the integral inequality

$$
x(t) \leq h+\int_{\pi_{1}}^{t} x(s) y(s) \mathrm{d} s, \quad t \in\left[\pi_{1}, \pi_{2}\right],
$$

then

$$
x(t) \leq h \exp \left(\int_{\pi_{1}}^{t} y(s) \mathrm{d} s, \quad t \in\left[\pi_{1}, \pi_{2}\right] .\right.
$$

Now, we give the following lemma to show the bounded of the solution for system (1):

Lemma 3. Assume that $\left(S\left(t_{0}\right), I_{1}\left(t_{0}\right), I_{2}\left(t_{0}\right), A\left(t_{0}\right)\right)$ is any initial value for system (1), then the region $\Omega=\left\{\left\{S(t), I_{1}(t), I_{2} \quad(t), A(t)\right\}: S(t) \geq 0, I_{1}(t) \geq 0\right.$, $\left.I_{2}(t) \geq 0, A(t) \geq 0, S(t)+I_{1}(t)+I_{2}(t)+A(t) \leq(\lambda / \mu)\right\}$ is a set of positive invariant.

Proof. From the equations of system (1), it follows that

$$
\begin{aligned}
{ }_{t_{0}}^{c} D_{t}^{\alpha}\left(S(t)+I_{1}(t)+I_{2}(t)+A(t)\right) & =\lambda-\mu\left(S(t)+I_{1}(t)+I_{2}(t)+A(t)\right)-a_{\sigma} I_{1}(t)-b_{\sigma} I_{2}(t)-c_{\sigma} A(t) \\
& \leq \lambda-\mu\left(S(t)+I_{1}(t)+I_{2}(t)+A(t)\right) .
\end{aligned}
$$

Based on the Laplace transform to (8), it follows that

$$
S(t)+I_{1}(t)+I_{2}(t)+A(t) \leq \frac{\lambda}{\mu} .
$$

So, $0 \leq S(t)+I_{1}(t)+I_{2}(t)+A(t) \leq \lambda / \mu$, which implies that $\Omega$ is a set of positive invariant for system (1).

Therefore, we will derive the threshold value of system (1) by calculating the spectral radius of a next generation integral operator and investigate global stability of $Q_{0}$ in the feasible region on the basis of the Lyapunov-Razumikhin method.

Theorem 1. Assume that $\left(T(t), I_{1}(t), I_{2}(t), A(t)\right)$ is a solution of system (1). Suppose that $\alpha \in(0,1)$ and

$$
\widehat{R}=\sup _{t \geq l} \frac{\int_{t_{0}}^{t} R_{\sigma}(\tau)(t-\tau)^{\alpha-1} \mathrm{~d} \tau}{\int_{t_{0}}^{t}(t-\tau)^{\alpha-1} \mathrm{~d} \tau}
$$

for some $l \geq t_{0}, \quad R_{\sigma}=p_{\sigma} \bar{S}\left(B_{\sigma}+E_{\sigma}\right) /\left(r_{\sigma}+a_{\sigma}+\mu\right) B_{\sigma}$, $B_{\sigma}=\left(p_{\sigma}+c_{\sigma}+\mu\right)\left(\phi_{\sigma}+b_{\sigma}+\mu\right)+\phi_{\sigma} \eta_{\sigma}-\phi_{\sigma} \eta_{\sigma} e_{\sigma} r_{\sigma}>0$, and $E_{\sigma}=\eta_{\sigma} r_{\sigma}\left(\phi_{\sigma}+e_{\sigma} b_{\sigma}+e_{\sigma} \mu\right)+e_{\sigma} r_{\sigma} q_{\sigma}\left(c_{\sigma}+\mu\right)>0$. If $\widehat{R}<1$, then the disease in system (1) dies out theoretically. In other words, the disease-free equilibrium $Q_{0}$ is globally asymptotically stable.

Proof. Construct the following set of Lyapunov functions:

$$
V(t)=X_{\sigma} I_{1}(t)+Y_{\sigma} I_{2}(t)+A(t)
$$

where $X_{\sigma}=\left[p_{\sigma}+c_{\sigma}+\mu+\eta_{\sigma} r_{\sigma}\left(1-e_{\sigma}\right)\right]\left(\phi_{\sigma}+b_{\sigma}+\mu\right)+$ $\left[e_{\sigma} r_{\sigma} q_{\sigma}\left(c_{\sigma}+\mu\right)+\phi_{\sigma} \eta_{\sigma}\right] / \eta_{\sigma}\left(r_{\sigma}+a_{\sigma}+\mu\right)\left(\phi_{\sigma}+b_{\sigma}+\mu\right)$ and $Y_{\sigma}=\left(c_{\sigma}+\mu\right) q_{\sigma}+\phi_{\sigma} \eta_{\sigma} / \eta_{\sigma}\left(\phi_{\sigma}+b_{\sigma}+\mu\right)$.

By Lemma 1 and taking the derivative of along system (1), we have

$$
\begin{aligned}
{ }_{t_{0}}^{c} D_{t}^{\alpha} V(t)= & X_{\sigma t_{0}}^{c} D_{t}^{\alpha} I_{1}(t)+Y_{\sigma t_{0}}^{c} D_{t}^{\alpha} I_{2}(t)+{ }_{t_{0}}^{c} D_{t}^{\alpha} A(t) \\
\leq & {\left[X_{\sigma} p_{\sigma} \bar{S}-X_{\sigma}\left(r_{\sigma}+a_{\sigma}+\mu\right)+Y_{\sigma} e_{\sigma} r_{\sigma}\right.} \\
& \left.+\left(1-e_{\sigma}\right) r_{\sigma}\right] I_{1}(t) \\
& +\left[X_{\sigma} q_{\sigma} \bar{S}-Y_{\sigma}\left(\phi_{\sigma}+b_{\sigma}+\mu\right)+\phi_{\sigma}\right] I_{2}(t) \\
& +\left[X_{\sigma} \eta_{\sigma} \bar{S}-\left(c_{\sigma}+\mu\right)\right] A(t) .
\end{aligned}
$$

Noting that $B_{\sigma}>0$ and $E_{\sigma}>0$, it follows that 


$$
\begin{aligned}
{ }_{t_{0}}^{c} D_{t}^{\alpha} V(t) \leq & G_{\sigma}\left(R_{\sigma}-1\right) I_{1}(t)+\frac{q_{\sigma} G_{\sigma}}{p_{\sigma}}\left(R_{\sigma}-1\right) I_{2}(t) \\
& +\frac{\eta_{\sigma} G_{\sigma}}{p_{\sigma}}\left(R_{\sigma}-1\right) A(t),
\end{aligned}
$$

in which $G_{\sigma}=X_{\sigma}\left(r_{\sigma}+a_{\sigma}+\mu\right)-Y_{\sigma} e_{\sigma} r_{\sigma}-\left(1-e_{\sigma}\right)$ and $R_{\sigma}=p_{\sigma} \bar{S}\left(B_{\sigma}+E_{\sigma}\right) /\left(r_{\sigma}+a_{\sigma}+\mu\right) B_{\sigma}$.

Letting $\widehat{T}=\max _{\sigma \in\{1,2, \ldots, m\}}\left\{1, q_{\sigma} / p_{\sigma}, \eta_{\sigma} / p_{\sigma}\right\}$, equation (13) can be written as

$$
{ }_{t_{0}}^{c} D_{t}^{\alpha} V(t) \leq \widehat{T} G_{\sigma}\left(R_{\sigma}-1\right)\left(I_{1}(t)+I_{2}(t)+A(t)\right) .
$$
have

On the contrary, taking $\hat{\theta}=\min _{\sigma \in\{1,2, \ldots, m\}}\left\{X_{\sigma}, Y_{\sigma}, 1\right\}$, we

$$
\begin{aligned}
\widehat{\theta}_{t_{0}}^{c} D_{t}^{\alpha}\left(I_{1}(t)+I_{2}(t)+A(t)\right) \leq & { }_{t_{0}}^{c} D_{t}^{\alpha} V(t) \\
\leq & \widehat{T} G_{\sigma}\left(R_{\sigma}-1\right)\left(I_{1}(t)+I_{2}(t)\right. \\
& +A(t)) .
\end{aligned}
$$

Combining equations (14) and (15), it follows that ${ }_{t_{0}}^{c} D_{t}^{\alpha}\left(I_{1}(t)+I_{2}(t)+A(t)\right) \leq \frac{\widehat{\theta}}{\widehat{T}} G_{\sigma}\left(R_{\sigma}-1\right)\left(I_{1}(t)+I_{2}(t)+A(t)\right)$.
Assume that $L(t)=I_{1}(t)+I_{2}(t)+A(t) \quad$ and $H=\max _{\sigma \in\{1,2, \ldots, m\}}\left\{\hat{\theta} / \widehat{T} G_{\sigma}\right\}$. Taking the fractional integral ${ }_{t_{0}}^{c} D_{t}^{-\alpha}$ on both sides of (16), for $t \in\left(t_{k-1}, t_{k}\right]$, we have

$$
L(t) \leq L\left(t_{k-1}\right)+\frac{H}{\Gamma(\alpha)} \int_{t_{k-1}}^{t}\left(R_{\sigma}-1\right)(t-\tau)^{\alpha-1} L(\tau) \mathrm{d} \tau .
$$

By Lemma 2, it leads to

$$
L(t) \leq L\left(t_{k-1}\right) \exp \left\{\frac{H}{\Gamma(\alpha)} \int_{t_{k-1}}^{t}\left(R_{\sigma}-1\right)(t-\tau)^{\alpha-1} \mathrm{~d} \tau\right\} .
$$

In addition, it follows that, for $t \in\left(0, t_{1}\right]$, $L(t) \leq L\left(t_{0}\right) \exp \left\{H / \Gamma(\alpha) \int_{t_{0}}^{t}\left(R_{\sigma}-1\right)(t-\tau)^{\alpha-1} \mathrm{~d} \tau\right\} . \quad$ Similarly, it can be shown that $t \in\left(t_{1}, t_{2}\right], L(t) \leq L\left(t_{1}\right) \exp$ $\left\{H / \Gamma(\alpha) \int_{t_{1}}^{t}\left(R_{\sigma}-1\right)(t-\tau)^{\alpha-1} \quad \mathrm{~d} \tau\right\} \leq L\left(t_{0}\right) \exp \{H / \Gamma(\alpha)$ $\left.\left[\int_{t_{0}}^{t_{1}}\left(R_{\sigma}-1\right)\left(t_{1}-\tau\right)^{\alpha-1} \mathrm{~d} \tau+\int_{t_{1}}^{t}\left(R_{\sigma}-1\right)(t-\tau)^{\alpha-1} \mathrm{~d} \tau\right]\right\}$.

Generally, for $t \in\left(t_{k-1}, t_{k}\right]$, it can be shown that

$$
L(t) \leq L\left(t_{0}\right) \exp \left\{H / \Gamma(\alpha)\left[\int_{t_{0}}^{t_{1}}\left(R_{\sigma}-1\right)\left(t_{1}-\tau\right)^{\alpha-1} \mathrm{~d} \tau+\cdots+\int_{t_{k-1}}^{t}\left(R_{\sigma}-1\right)(t-\tau)^{\alpha-1} \mathrm{~d} \tau\right]\right\} .
$$

Since $\widehat{R}<1$, it can be deducted that $\zeta=\exp \left\{H / \Gamma(\alpha)\left[\int_{t_{0}}^{t_{1}}\right.\right.$ $\left.\left.\left(R_{\sigma}-1\right)\left(t_{1}-\tau\right)^{\alpha-} \quad 1 \mathrm{~d} \tau+\cdots+\int_{t_{k-1}}^{t}\left(R_{\sigma}-1\right)(t-\tau)^{\alpha-1} \mathrm{~d} \tau\right]\right\}<1$. Note that $I_{1}, I_{2}, A \geq 0$, and $I_{1}, I_{2}^{k-1}$, and $A$ converge to zero exponentially, and hence, $S$ approaches $\bar{S}$. In other words, the disease in system (1) dies out theoretically.

Remark 1. For all $\sigma \in\{1,2, \ldots, m\}, R_{\sigma}<1$, and Theorem 1 is satisfied. This is because, for $\tau \in\left(t_{k-1}, t\right), t-\tau>0$, and it follows that $(t-\tau)^{\alpha-1}>0$ and $\int_{t_{0}}^{t_{1}}\left(R_{\sigma}-1\right)\left(t_{1}-\right.$ $\tau)^{\alpha-1} \mathrm{~d} \tau+\cdots+\int_{t_{k-1}}^{t}\left(R_{\sigma}-1\right)(t-\tau)^{\alpha-1} \mathrm{~d} \tau<0$, which leads to $I_{1}, I_{2}$, and $A$ converge to zero, and $S$ approaches $\bar{S}$.

Next, biological and environmental parameters are periodic variation due to the seasonal changes, and we will assume that a switching rule $\sigma$ for system (1) is periodic and satisfies the following conditions [35]: $\omega$ denotes one switching period, and $t_{k}-t_{k-1}=\omega_{k}$ with $\omega_{k+m}=\omega_{k}$ and $\omega=\omega_{1}+\cdots+\omega_{m}$. Take $\mathscr{I}_{\text {periodic }}$ be the set of periodic switching rule and $\mathscr{I}_{\text {periodic }} \subset \mathscr{I}$. Thus, the following results are presented.

Theorem 2. Assume that $\left(T(t), I_{1}(t), I_{2}(t), A(t)\right)$ is a solution of system (1). Assume that the switching rule $\sigma$ is periodic. Suppose that $\alpha \in(0,1)$ and

$$
\bar{R}=\frac{R_{1} \omega_{1}^{\alpha}+R_{2} \omega_{2}^{\alpha}+\cdots+R_{m} \omega_{m}^{\alpha}}{\omega_{1}^{\alpha}+\omega_{2}^{\alpha}+\ldots \omega_{m}^{\alpha}}
$$

in which, for $\sigma \in\{1,2, \ldots, m\}, \quad R_{\sigma}=p_{\sigma} \bar{S}\left(B_{\sigma}+E_{\sigma}\right) /$ $\left(r_{\sigma}+a_{\sigma}+\mu\right) B_{\sigma}, \quad B_{\sigma}=\left(p_{\sigma}+c_{\sigma}+\mu\right)\left(\phi_{\sigma}+b_{\sigma}+\mu\right)+$ $\phi_{\sigma} \eta_{\sigma}-\phi_{\sigma} \eta_{\sigma} e_{\sigma} r_{\sigma}>0$, and $E_{\sigma}=\eta_{\sigma} r_{\sigma}\left(\phi_{\sigma}+e_{\sigma} b_{\sigma}+e_{\sigma} \mu\right)+$ $e_{\sigma} r_{\sigma} q_{\sigma}\left(c_{\sigma}+\mu\right)$. If $\bar{R}<1$, then the disease in system (1) dies out theoretically. In other words, the disease-free equilibrium $Q_{0}$ is globally asymptotically stable.

Proof. Assume that the switching rule $\sigma$ is periodic. By the proof of Theorem 1, for $t=t_{0}+\omega$, it follows that

$$
L\left(t_{0}+\omega\right) \leq L\left(t_{0}\right) \exp \left\{\frac{H}{\Gamma(\alpha)}\left[\int_{t_{0}}^{t_{1}}\left(R_{\sigma}-1\right)\left(t_{1}-\tau\right)^{\alpha-1} d \tau+\cdots+\int_{t_{m-1}}^{t_{m}}\left(R_{\sigma}-1\right)\left(t_{m}-\tau\right)^{\alpha-1} d \tau\right]\right\} \leq L\left(t_{0}\right) \zeta
$$


in which $\zeta=\exp \left\{H / \Gamma(\alpha)\left[\int_{t_{0}}^{t_{1}}\left(R_{\sigma}-1\right)\left(t_{1}-\tau\right)^{\alpha-1} \mathrm{~d} \tau+\cdots\right.\right.$ $\left.\left.+\int_{t_{m-1}}^{t_{m}}\left(R_{\sigma}-1\right)\left(t_{m}-\tau\right)^{\alpha-1} \mathrm{~d} \tau\right]\right\}$. According to the condition of $\bar{R}<1$, we can get $\zeta<1$. For some integer $h=1,2, \ldots$, $L\left(t_{0}+h \omega\right) \leq \zeta L\left(t_{0}+\quad(h-1) \omega\right) \leq \ldots \leq \zeta^{h} L\left(t_{0}\right)$. The sequence $\left\{L\left(t_{0}+h \omega\right)\right\} \longrightarrow 0, h \longrightarrow \infty$. In general, for $t \in\left(t_{k-1}, t_{k}\right]$ and $t_{0}+h \omega<t_{k} \leq t_{0}+(h+1) \omega$, it follows that $L(t) \leq L\left(t_{0}+h \omega\right) \exp \left\{H / \Gamma(\alpha) \int_{t_{0}+h \omega}^{t}\left(R_{\sigma}-1\right) \quad(t-\tau)^{\alpha-1}\right.$ $\mathrm{d} \tau\} \leq M L\left(t_{0}+h \omega\right)$, where $\quad M=\max _{t_{0}+h \omega<t \leq t_{0}+(h+1) \omega}$ $\exp \left\{H / \Gamma(\alpha) \int_{t_{0}+h \omega}^{t}\left(R_{\sigma}-1\right)(t-\tau)^{\alpha-1} \mathrm{~d} \tau\right\}$. By the facts that $I_{1}, I_{2}, A \geq 0$, we can deduct that $I_{1}, I_{2}$, and $A$ converge to zero, and hence, $S$ approaches $\bar{S}$, which means that the disease in system (1) dies out theoretically.

Remark 2. Even though threshold value $R_{\sigma}$ of some subsystems in system (1) is greater than one, $Q_{0}$ is globally asymptotically stable as long as threshold value $\bar{R}$ of system (1) is less than one.

\section{Treatment Strategies}

In this section, we incorporate a treatment compartment $U(t)$ into the fractional order HIV/AIDS model with switching parameters. Assume that infected individuals $I_{1}(t)$ with asymptomatic receive drug treatment and the proportion of effective treatment is $\tau_{1}$, infected individuals $I_{2}(t)$ with symptomatic receive drug treatment and the proportion of effective treatment is $\tau_{2}$, and individuals diagnosed with AIDS $A(t)$ receive drug treatment and the proportion of effective treatment is $\tau_{3}$. Therefore, a new fractional order HIV/AIDS model with switching parameters and treatment compartment can be written as follows:

$$
\left\{\begin{array}{l}
{ }_{t_{0}}^{c} D_{t}^{\alpha} S(t)=\lambda-p_{\sigma} S(t) I_{1}(t)-q_{\sigma} S(t) I_{2}(t)-\eta_{\sigma} S(t) A(t)-\mu S(t), \\
{ }_{t_{0}}^{c} D_{t}^{\alpha} I_{1}(t)=p_{\sigma} S(t) I_{1}(t)+q_{\sigma} S(t) I_{2}(t)+\eta_{\sigma} S(t) A(t)-\left(r_{\sigma}+a_{\sigma}+\mu\right) I_{1}(t)-\tau_{1} I_{1}(t), \\
{ }_{t_{0}} D_{t}^{\alpha} I_{2}(t)=e_{\sigma} r_{\sigma} I_{1}(t)-\left(\phi_{\sigma}+b_{\sigma}+\mu\right) I_{2}(t)-\tau_{2} I_{2}(t), \\
{ }^{c} D_{t}^{\alpha} A(t)=\left(1-e_{\sigma}\right) r_{\sigma} I_{1}(t)+\phi_{\sigma} I_{2}(t)-\left(c_{\sigma}+\mu\right) A(t)-\tau_{3} A(t), \\
{ }_{t_{0}}^{c} D_{t}^{\alpha} U(t)=\tau_{1} I_{1}(t)+\tau_{2} I_{2}(t)+\tau_{3} A(t)-\left(d_{\sigma}+\mu\right) U(t)
\end{array}\right.
$$

According to Definition 4 , the disease-free equilibrium of system (22) is $E_{0}=(\bar{S}, 0,0,0,0)$, in which $\bar{S}=\lambda / \mu$. For any initial value $\left(S\left(t_{0}\right), I_{1}\left(t_{0}\right), I_{2}\left(t_{0}\right), A\left(t_{0}\right), U\left(t_{0}\right)\right)$ of system (22), the region $\Omega=\left\{\left(S(t), I_{1}(t), I_{2}(t), \quad A(t), U(t)\right)\right.$ : $S(t) \geq 0, I_{1}(t) \geq 0, I_{2}(t) \geq 0, A(t) \geq 0, S(t)+I_{1}(t)+I_{2}(t)+$ $A(t)+U(t) \leq \lambda / \mu\}$ is a positive invariant set. In the following, we analysis the global asymptotical stability of $E_{0}$ for system (22).

Theorem 3. Assume that $\left(T(t), I_{1}(t), I_{2}(t), A(t), U(t)\right)$ is a solution of system (22). Suppose that $\alpha \in(0,1)$ and

$$
\widetilde{R}=\sup _{t \geq l} \frac{\int_{t_{0}}^{t} \chi_{\sigma}(\tau)(t-\tau)^{\alpha-1} \mathrm{~d} \tau}{\int_{t_{0}}^{t}(t-\tau)^{\alpha-1} \mathrm{~d} \tau},
$$

for some $l \geq t_{0}, \chi_{\sigma}=p_{\sigma} \bar{S}\left[p_{\sigma}\left(c_{\sigma}+\mu+\tau_{3}\right)+\eta_{\sigma} G_{\sigma}\right] / p_{\sigma}\left(c_{\sigma}+\mu+\right.$ $\left.\tau_{3}\right)+\eta_{\sigma} G_{\sigma}-\left(r_{\sigma}+a_{\sigma}+\mu+\tau_{1}\right) \eta_{\sigma} G_{\sigma}>0$ and $G_{\sigma}=\left[\left(c_{\sigma}+\mu+\right.\right.$ $\left.\left.\tau_{3}\right) q_{\sigma}+\phi_{\sigma} \eta_{\sigma}\right]\left(1-e_{\sigma}\right) e_{\sigma} r_{\sigma}^{2}$. If $\widetilde{R}<1$, then the disease in system (22) dies out theoretically. In other words, the disease-free equilibrium $E_{0}$ is globally asymptotically stable.

Proof. Construct the following set of Lyapunov functions for system (22):

$$
\mathrm{F}(t)=\xi_{\sigma} I_{1}(t)+\psi_{\sigma} I_{2}(t)+A(t)
$$

where $\xi_{\sigma}=p_{\sigma}\left(c_{\sigma}+\mu+\tau_{3}\right)+\eta_{\sigma} G_{\sigma} /\left(r_{\sigma}+a_{\sigma}+\mu+\tau_{1}\right) \eta_{\sigma}$ and $\psi_{\sigma}=\left(c_{\sigma}+\mu+\tau_{3}\right) q_{\sigma}+\phi_{\sigma} \eta_{\sigma} /\left(\phi_{\sigma}+b_{\sigma}+\mu+\tau_{2}\right) \eta_{\sigma}$.

According to system (22), we have

$$
\begin{aligned}
{ }_{t_{0}}^{c} D_{t}^{\alpha} \mathrm{F}(t)= & \xi_{\sigma t_{0}}^{c} D_{t}^{\alpha} I_{1}(t)+\psi_{\sigma t_{0}}^{c} D_{t}^{\alpha} I_{2}(t)+{ }_{t_{0}}^{c} D_{t}^{\alpha} A(t) \\
\leq & {\left[\xi_{\sigma} p_{\sigma} \bar{S}-\xi_{\sigma}\left(r_{\sigma}+a_{\sigma}+\mu+\tau_{1}\right)+\psi_{\sigma} e_{\sigma} r_{\sigma}+\left(1-e_{\sigma}\right) r_{\sigma}\right] I_{1}(t)+\left[\xi_{\sigma} q_{\sigma} \bar{S}-\psi_{\sigma}\left(\phi_{\sigma}+b_{\sigma}+\mu+\tau_{2}\right)+\phi_{\sigma}\right] I_{2}(t) } \\
& +\left[\xi_{\sigma} \eta_{\sigma} \bar{S}-\left(c_{\sigma}+\mu+\tau_{3}\right)\right] A(t) \leq \overline{G_{\sigma}}\left(\chi_{\sigma}-1\right) I_{1}(t) \\
& +\frac{q_{\sigma} \overline{G_{\sigma}}}{p_{\sigma}}\left(\chi_{\sigma}-1\right) I_{2}(t)+\frac{\eta_{\sigma} \overline{G_{\sigma}}}{p_{\sigma}}\left(\chi_{\sigma}-1\right) A(t)
\end{aligned}
$$

in which $\overline{G_{\sigma}}=p_{\sigma}\left(c_{\sigma}+\mu+\tau_{3}\right)+\left(1-r_{\sigma}-a_{\sigma}-\mu /\right.$ $\operatorname{tau}_{1} \eta_{\sigma} G_{\sigma} r_{\sigma}+a_{\sigma}+\mu+\tau_{1} \quad$ and $\quad \chi_{\sigma}=p_{\sigma} \bar{S}\left[p_{\sigma}\left(c_{\sigma}+\mu+\right.\right.$ $\left.\left.\tau_{3}\right)+\eta_{\sigma} G_{\sigma}\right] / p_{\sigma}\left(c_{\sigma}+\mu+\tau_{3}\right)+\eta_{\sigma} G_{\sigma}-\left(r_{\sigma}+a_{\sigma}+\mu+\right.$ $\left.\tau_{1}\right) \eta_{\sigma} G_{\sigma}>0$. 
Letting $\bar{T}=\max _{\sigma \in\{1,2, \ldots, m\}}\left\{1, q_{\sigma} / p_{\sigma}, \eta_{\sigma} / p_{\sigma}\right\}$, we can obtain ${ }_{t_{0}}^{c} D_{t}^{\alpha} \mathrm{F}(t) \leq \overline{T G_{\sigma}}\left(\chi_{\sigma}-1\right)\left(I_{1}(t)+I_{2}(t)+A(t)\right)$.
Taking $\bar{\theta}=\min _{\sigma \in\{1,2, \ldots, m\}}\left\{\xi_{\sigma}, \psi_{\sigma}, 1\right\}$ and combining equations (23) and (26), we have

$$
\bar{\theta}_{t_{0}}^{c} D_{t}^{\alpha}\left(I_{1}(t)+I_{2}(t)+A(t)\right) \leq_{t_{0}}^{c} D_{t}^{\alpha} \mathrm{F}(t) \leq \overline{T G_{\sigma}}\left(\chi_{\sigma}-1\right)\left(I_{1}(t)+I_{2}(t)+A(t)\right) .
$$

It follows that

$$
\begin{aligned}
&{ }_{t_{0}}^{c} D_{t}^{\alpha}\left(I_{1}(t)+I_{2}(t)+A(t)\right) \leq \overline{\bar{T}} \\
& \overline{G_{\sigma}}\left(\chi_{\sigma}-1\right)\left(I_{1}(t)+I_{2}(t)\right. \\
&+A(t)) .
\end{aligned}
$$

Letting $\quad \overline{L(t)}=I_{1}(t)+I_{2}(t)+A(t)$ and $\bar{H}=\max _{\sigma \in\{1,2, \ldots, m\}}\left\{\bar{\theta} / \overline{T G_{\sigma}}\right\}$ and taking the fractional integral ${ }_{t_{0}}^{c} D_{t}^{-\alpha}$ to both sides of (28), for $t \in\left(t_{k-1}, t_{k}\right]$, it follows that

$$
\overline{L(t)} \leq \overline{L\left(t_{k-1}\right)}+\frac{\bar{H}}{\Gamma(\alpha)} \int_{t_{k-1}}^{t}\left(\chi_{\sigma}-1\right)(t-\tau)^{\alpha-1} \overline{L(\tau)} \mathrm{d} \tau .
$$

Furthermore, we derive

$$
\overline{L(t)} \leq \overline{L\left(t_{k-1}\right)} \exp \left\{\frac{\bar{H}}{\Gamma(\alpha)} \int_{t_{k-1}}^{t}\left(\chi_{\sigma}-1\right)(t-\tau)^{\alpha-1} \mathrm{~d} \tau\right\} .
$$

Then, it follows that, for $t \in\left(0, t_{1}\right], \quad \overline{L(t)} \leq$ $\overline{L\left(t_{0}\right)} \exp \left\{\bar{H} / \Gamma(\alpha) \int_{t_{0}}^{t}\left(\chi_{\sigma}-1\right)(t-\tau)^{\alpha-1} \mathrm{~d} \tau\right\}$. Similarly, it can be shown that $t \in\left(t_{1}, t_{2}\right], \overline{L(t)} \leq \overline{L\left(t_{1}\right)} \exp \left\{\bar{H} / \Gamma(\alpha) \int_{t_{1}}^{t}\left(\chi_{\sigma}-\right.\right.$ 1) $\left.(t-\tau)^{\alpha-1} \mathrm{~d} \tau\right\} \leq \overline{L\left(t_{0}\right)} \exp \quad\left\{\bar{H} / \Gamma(\alpha)\left[\int_{t_{0}}^{t_{1}}\left(\chi_{\sigma}-1\right)\right.\right.$ $\left.\left.\left(t_{1}-\tau\right)^{\alpha-1} \mathrm{~d} \tau+\int_{t_{1}}^{t}\left(\chi_{\sigma}-1\right) \quad(t-\tau)^{\alpha-1} \mathrm{~d} \tau\right]\right\}$. Generally, for $t \in\left(t_{k-1}, t_{k}\right]$, it can be shown that

$$
\overline{L(t)} \leq \overline{L\left(t_{0}\right)} \exp \left\{\frac{\bar{H}}{\Gamma(\alpha)}\left[\int_{t_{0}}^{t_{1}}\left(\chi_{\sigma}-1\right)\left(t_{1}-\tau\right)^{\alpha-1} \mathrm{~d} \tau+\cdots+\int_{t_{k-1}}^{t}\left(\chi_{\sigma}-1\right)(t-\tau)^{\alpha-1} \mathrm{~d} \tau\right]\right\} .
$$

Since $\widetilde{R}<1$, it can be obtained that $\bar{\zeta}=\exp$ $\left\{\bar{H} / \Gamma(\alpha)\left[\int_{t_{0}}^{t_{1}}\left(\chi_{\sigma}-1\right)\left(t_{1}-\tau\right)^{\alpha-1} \mathrm{~d} \tau+\cdots+\int_{t_{k-1}}^{t}\left(\chi_{\sigma}-1\right)(t-\right.\right.$ $\left.\left.\tau)^{\alpha-1} \mathrm{~d} \tau\right]\right\}<1$. Note that $I_{1}, I_{2}, A, U \geq 0$, and hence, $I_{1}, I_{2}, A$, and $U$ converge to zero exponentially, and $S$ approaches $\bar{S}$. Therefore, the disease in system (22) dies out theoretically.

In addition, we study the dynamics of system (22) when the switching rule is periodic. The following results are given.

Theorem 4. Assume that (T(t), $\left.I_{1}(t), I_{2}(t), A(t), U(t)\right)$ is a solution of system (22). Assume that the switching rule $\sigma$ is periodic. Suppose that $\alpha \in(0,1)$ and

$$
R^{*}=\frac{\chi_{1} \omega_{1}^{\alpha}+\chi_{2} \omega_{2}^{\alpha}+\cdots+\chi_{m} \omega_{m}^{\alpha}}{\omega_{1}^{\alpha}+\omega_{2}^{\alpha}+\ldots \omega_{m}^{\alpha}}
$$

in which, for $\sigma \in\{1,2, \ldots, m\}, \chi_{\sigma}=p_{\sigma} \bar{S}\left[p_{\sigma}\left(c_{\sigma}+\mu+\tau_{3}\right)+\right.$ $\left.\eta_{\sigma} G_{\sigma}\right] / p_{\sigma}\left(c_{\sigma}+\mu+\tau_{3}\right)+\eta_{\sigma} G_{\sigma}-\left(r_{\sigma}+a_{\sigma}+\mu+\tau_{1}\right) \eta_{\sigma} G_{\sigma}>0$ and $G_{\sigma}=\left[\left(c_{\sigma}+\mu+\tau_{3}\right) q_{\sigma}+\phi_{\sigma} \eta_{\sigma}\right]\left(1-e_{\sigma}\right) e_{\sigma} r_{\sigma}^{2}$. If $R^{*}<1$, then the disease in system (22) dies out theoretically. In other words, the disease-free equilibrium $E_{0}$ is globally asymptotically stable.

Proof. Assume that the switching rule $\sigma$ is periodic. By the proof of Theorem 3, it follows that, for $t=t_{0}+\omega$,

$$
\begin{aligned}
\overline{L\left(t_{0}+\omega\right)} & \leq \overline{L\left(t_{0}\right)} \exp \left\{\frac{H}{\Gamma(\alpha)}\left[\int_{t_{0}}^{t_{1}}\left(\chi_{\sigma}-1\right)\left(t_{1}-\tau\right)^{\alpha-1} \mathrm{~d} \tau+\cdots+\int_{t_{m-1}}^{t_{m}}\left(\chi_{\sigma}-1\right)\left(t_{m}-\tau\right)^{\alpha-1} \mathrm{~d} \tau\right]\right\} \\
& \leq L\left(t_{0}\right) \zeta
\end{aligned}
$$

in which $\zeta^{*}=\exp \left\{H / \Gamma(\alpha)\left[\int_{t_{0}}^{t_{1}}\left(\chi_{\sigma}-1\right)\left(t_{1}-\tau\right)^{\alpha-1} \mathrm{~d} \tau+\cdots+\right.\right.$ $\left.\left.\int_{t_{m-1}}^{t_{m}}\left(\chi_{\sigma}-1\right)\left(t_{m}-\tau\right)^{\alpha-1} \mathrm{~d} \tau\right]\right\}$. Noting that $R^{*}<1$, we can get $\zeta^{*}<1 . \quad$ For some integer $h=1,2, \ldots, \quad \overline{L\left(t_{0}+h \omega\right)} \leq$ $\zeta \overline{L\left(t_{0}+(h-1) \omega\right)} \leq \ldots \leq \zeta^{* h} \overline{L\left(t_{0}\right)} . \quad$ The sequence $\left\{\overline{L\left(t_{0}+h \omega\right)}\right\} \longrightarrow 0, h \longrightarrow \infty$. In general, for $t \in\left(t_{k-1}, t_{k}\right]$ and $t_{0}+h \omega<t_{k} \leq t_{0}+(h+1) \omega, \quad$ it follows that $\overline{L(t)} \leq \overline{L\left(t_{0}+h \omega\right)} \exp \left\{H / \quad \Gamma(\alpha) \int_{t_{0}+h \omega}^{t}\left(\chi_{\sigma}-1\right) \quad(t-\tau)^{\alpha-1}\right.$ 


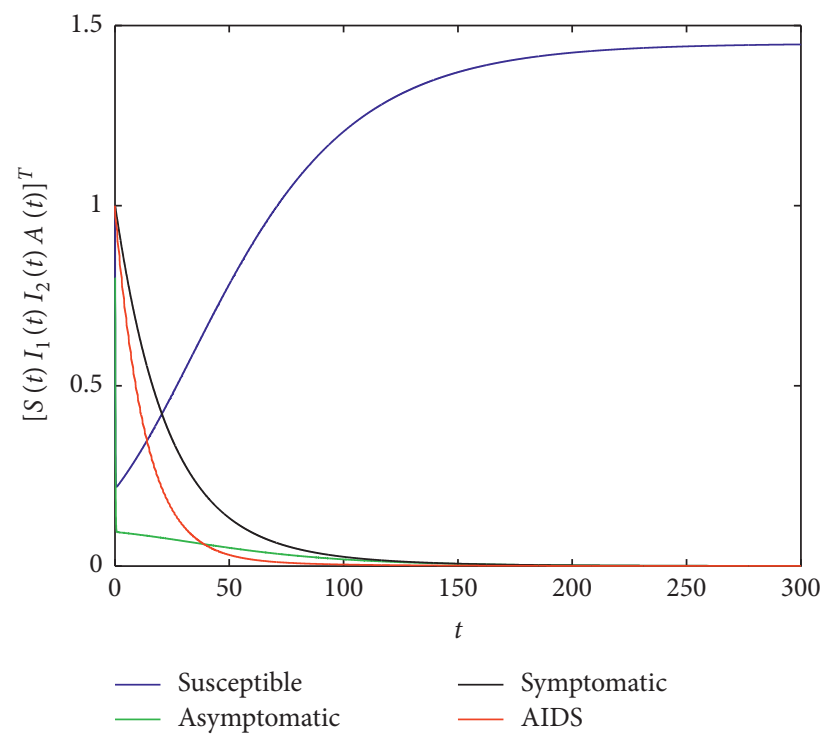

FIgURE 1: The solutions of system (1) with the fractional order $\alpha=0.065$.

$\mathrm{d} \tau\} \leq \bar{M} \overline{L\left(t_{0}+h \omega\right)}$, where $\bar{M}=\max _{t_{0}+h \omega<t \leq t_{0}+(h+1) \omega} \exp \{H /$ $\left.\Gamma(\alpha) \int_{t_{0}+h \omega}^{t}\left(\chi_{\sigma}-1\right)(t-\tau)^{\alpha-1} \mathrm{~d} \tau\right\}$. Note that $I_{1}, I_{2}, A, U \geq 0$, and hence, $I_{1}, I_{2}, A$, and $U$ converge to zero exponentially, and $S$ approaches $\bar{S}$. Therefore, the disease in system (22) dies out theoretically.

\section{Numerical Simulations}

In this section, numerical examples are presented to substantiate our theoretical results introduced. Suppose that $t_{0}=0, m=2$, and one period is $\omega=1$ with $\omega_{1}=0.9$ and $\omega_{2}=0.1$. Most of the parameter values are taken from those in $[2,7,8,17]$. Assume that constant parameter values are $\lambda=0.029$ and $u=0.02$. Take the switching parameters $p_{1}=0.064, q_{1}=0.07, r_{1}=0.05$, $\eta_{1}=0.01, e_{1}=0.6, \quad b_{1}=0.02, \quad a_{1}=0.1$, $\varphi_{1}=0.001, c_{1}=0.33, \quad p_{2}=0.09, \quad q_{2}=0.087, \quad r_{2}=0.04$, $\eta_{2}=0.02, e_{2}=0.7, b_{2}=0.04, a_{2}=0.2$, and $\varphi_{2}=0.001$ with initial values $S(0)=1.0, I_{1}(0)=0.80, I_{2}(0)=1.0$, and $A(0)=1.0$. Taking the fractional order parameter $\alpha=0.065$, it follows that the threshold value in the $1 s t$ subsystem is 1.6087; the threshold value in the 2 nd subsystem is 0.2335 . It can be calculated $\bar{R}=0.8720<1$ by Theorem 2. Figure 1 shows that the disease can be cleared under the condition of $\bar{R}<1$, which sustains the result given in by Theorem 2. Moreover, when taking a different fractional order $\alpha$, Figure 2 describes the individuals behaviors. It is observed from Figure 2 that the dynamics of the asymptomatic infected individuals and the symptomatic infected individuals increase as the fractional order increases, but the susceptible individuals decrease. Therefore, the fractional order and switching parameters have a great impact on the disease eradication.

On the contrary, take the treatment parameter $\tau_{1}=\tau_{2}=\tau_{3}=0.1$, and $U(0)=1.0$. The other parameters are the same as Figure 1. It can be obtained that $R^{*}=$ $0.1259<1$ by Theorem 4 . Figure 3 shows that the disease dies out when $R^{*}<1$, which consists of the result given in by Theorem 4. Moreover, Figure 4 describes the individuals' behaviors with a different fractional order $\alpha$. Compared with the higher values of the fractional order, it can be found that the treatment is more effective when taking small value. 

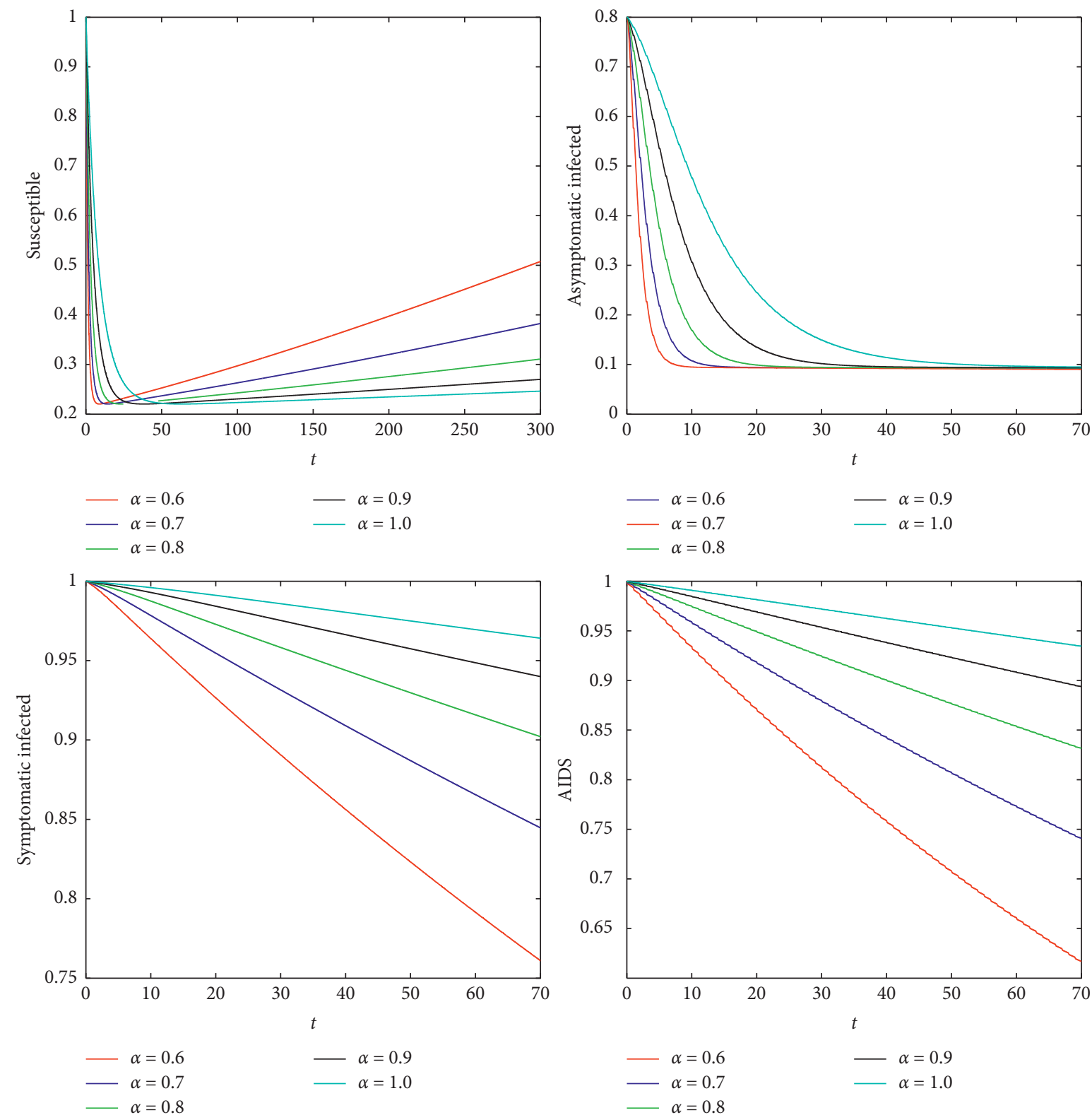

Figure 2: The dynamics of system (1) for different fractional order $\alpha$.

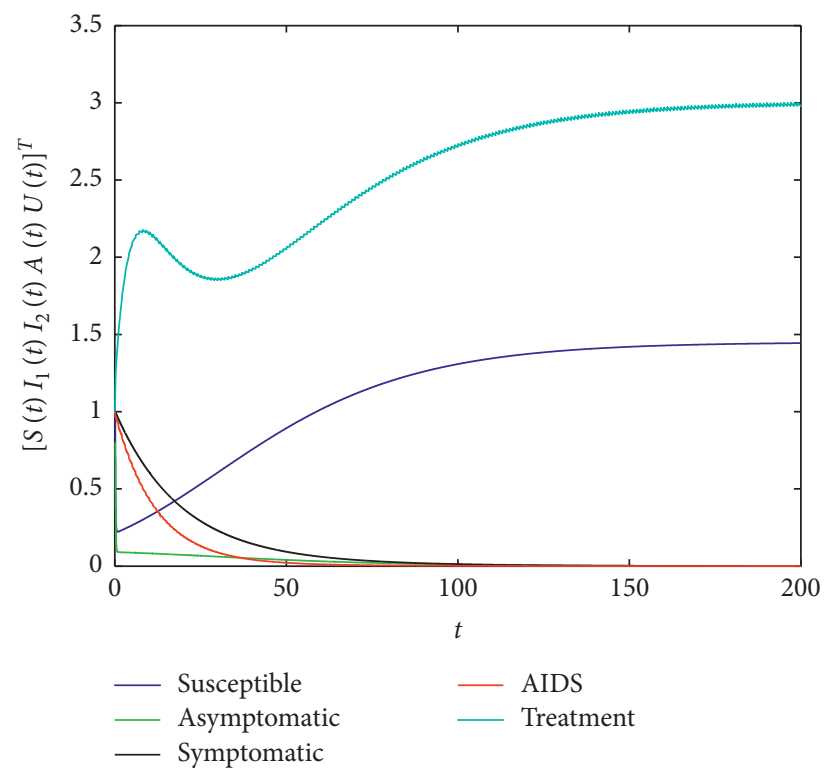

FIgURE 3: The solutions of system (22) with the fractional order $\alpha=0.065$. 

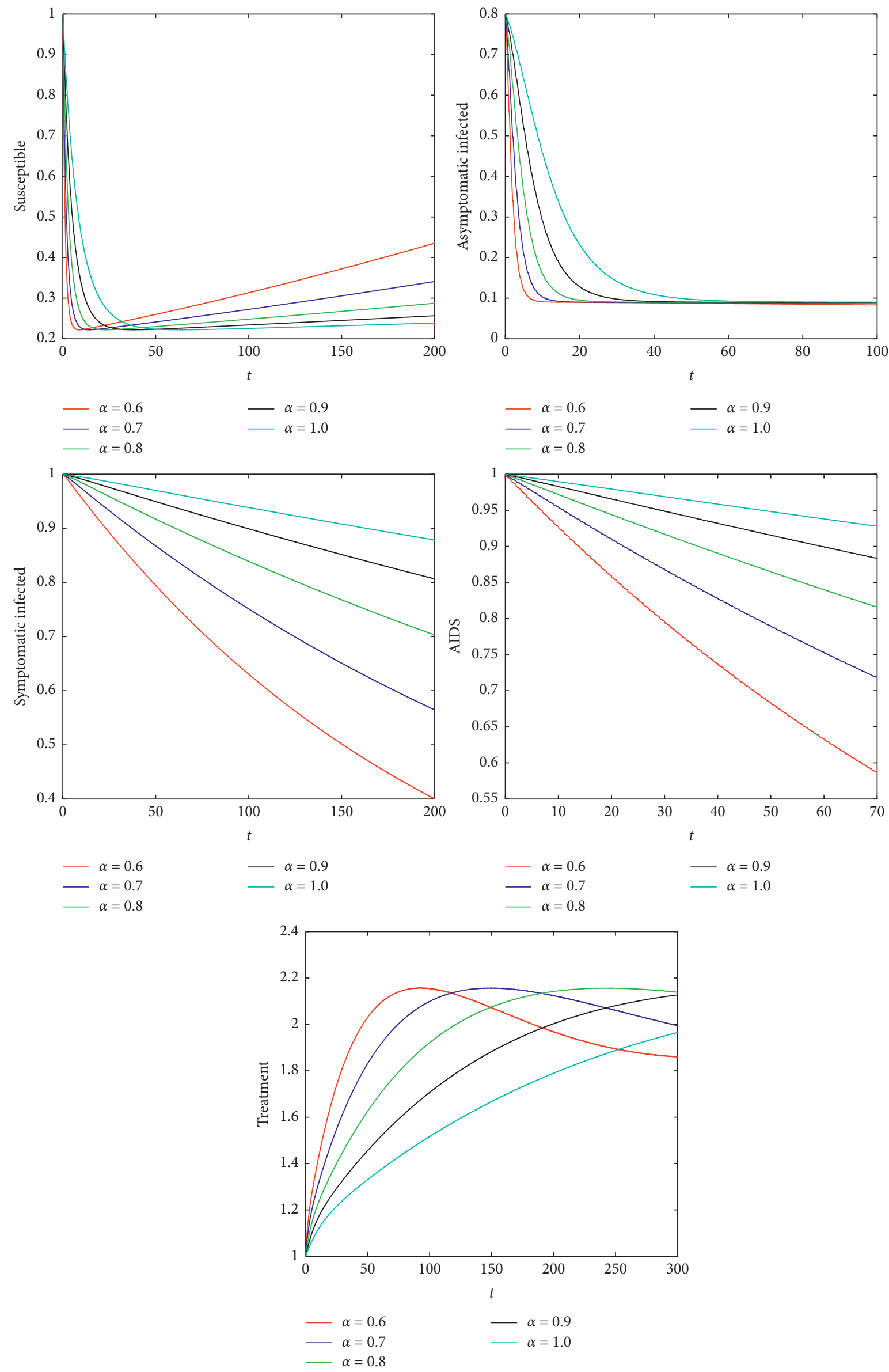

Figure 4: The dynamics of system (22) for different fractional order $\alpha$. 


\section{Conclusions}

Research on HIV/AIDS epidemic models involving fractional order derivative $0<\alpha<1$, switching parameters, and treatment compartment are becoming one of the key areas in mathematical theory of epidemiology. As we all known, the research works on the switched HIV/AIDS epidemic models are very few [36-38]. This paper has studied the global asymptotical stability of new fractional order HIV/AIDS models with switching parameters and treatment compartment. By constructing Lyapunov-Razumikhin technique, new threshold values are obtained to ensure eradication of the disease. More specifically, when $\widehat{R}<1$ or $\bar{R}<1$, the disease-free equilibrium $Q_{0}$ of system (1) is globally asymptotically stable; in other words, the disease in system (1) dies out theoretically. Moreover, it has been shown that fractional order and switching parameters are related to threshold values, which have a significant effect on extinction of disease. On the contrary, when incorporating treatment compartments into the fractional order HIV/ AIDS model with switching parameters, a new HIV/AIDS model is developed. The obtained results show that the disease can be cleared theoretically when the threshold value is less than one. In addition, numerical results show when the fractional order increases, the dynamics of the asymptomatic infected individuals and the symptomatic infected individuals increase while the susceptible individuals decrease. One future work is to investigate the endemic case for fractional order switched HIV/AIDS models.

\section{Data Availability}

The data used to support the findings of this study are included within the article.

\section{Conflicts of Interest}

The authors declare that there are no conflicts of interest regarding the publication of this paper.

\section{Acknowledgments}

This work was supported by the National Natural Science Foundation of China (Grant no. 11847081), Key Scientific Research Projects of Henan Province (Grant nos. 17A110039 and 19A110007), Fundamental Research Funds for the Henan Provincial Colleges and Universities in Henan University of Technology (Grant no. 2017QNJH18), and High-Level Personal Foundation of Henan University of Technology (no. 2017BS009).

\section{References}

[1] M. Kgosimore and E. M. Lungu, "The effects of vaccination and treatment on the spread of HIV/AIDS," Journal of Biological Systems, vol. 12, no. 4, pp. 399-417, 2004.

[2] R. M. Anderson, G. F. Medley, R. M. May, and A. M. Johnson, "A preliminary study of the transmission dynamics of the Human Immunodeficiency Virus (HIV), the causative agent of AIDS," Mathematical Medicine and Biology, vol. 3, no. 4, pp. 229-263, 1986.

[3] B. Schwartlander, J. Stover, N. Walker et al., "Resource needs for HIV/AIDS," Science, vol. 292, pp. 2434-2436, 2001.

[4] D. Trono, C. Van Lint, C. Rouzioux et al., "HIV persistence and the prospect of long-term drug-free remissions for HIV-infected individuals," Science, vol. 329, no. 5988, pp. 174-180, 2010.

[5] M. U. Nsuami and P. J. Witbooi, "Stochastic dynamics of an HIV/AIDS epidemic model with treatment," Quaestiones Mathematicae, vol. 42, no. 5, pp. 605-621, 2019.

[6] K. O. Okosun, O. D. Makinde, and I. Takaidza, "Impact of optimal control on the treatment of HIV/AIDS and screening of unaware infectives," Applied Mathematical Modelling, vol. 37, no. 6, pp. 3802-3820, 2013.

[7] M. Pitchaimani and C. Monica, "Global stability analysis of HIV-1 infection model with three time delays," Journal of Applied Mathematics and Computing, vol. 48, no. 1-2, pp. 293-319, 2015.

[8] C. J. Silva and D. F. M. Torres, "Stability of a fractional HIV/ AIDS model," Mathematics and Computers in Simulation, vol. 164, pp. 180-190, 2019.

[9] J. Huo, H. Zhao, and L. Zhu, "The effect of vaccines on backward bifurcation in a fractional order HIV model," Nonlinear Analysis: Real World Applications, vol. 26, pp. 289-305, 2015.

[10] C. Wu and X. Liu, "Lyapunov and external stability of Caputo fractional order switching systems," Nonlinear Analysis: Hybrid Systems, vol. 34, pp. 131-146, 2019.

[11] H. Liu, Y. Pan, S. Li, and Y. Chen, "Adaptive fuzzy backstepping control of fractional-order nonlinear systems," IEEE Transactions on Systems, Man, and Cybernetics: Systems, vol. 47, no. 8, pp. 2209-2217, 2017.

[12] Y. Li, Y. Chen, and I. Podlubny, "Mittag-Leffler Stability of fractional order nonlinear dynamic systems," Automatica, vol. 45, no. 8, pp. 1965-1969, 2009.

[13] F. A. Rihan, Q. M. Al-Mdallal, H. J. AlSakaji, and A. Hashish, "A fractional-order epidemic model with time-delay and nonlinear incidence rate," Chaos, Solitons \& Fractals, vol. 126, pp. 97-105, 2019.

[14] M. Saeedian, M. Khalighi, N. Azimi Tafreshi, G. R. Jafari, and M. Ausloos, "Memory effects on epidemic evolution: the susceptible infected recovered epidemic model," Physical Review E, vol. 95, no. 2, p. 022409, 2017.

[15] E. Okyere, F. Oduro, S. Amponsah, I. Dontwi, and N. Frempong, "Fractional order SIR model with constant population," British Journal of Mathematics \& Computer Science, vol. 14, no. 2, pp. 1-12, 2016.

[16] S. Ullah, M. Altaf Khan, M. Altaf Khan, M. Farooq, Z. Hammouch, and D. Baleanu, "A fractional model for the dynamics of tuberculosis infection using Caputo-Fabrizio derivative," Discrete \& Continuous Dynamical Systems-S, vol. 13, no. 3, pp. 975-993, 2020.

[17] H. Kheiri and M. Jafari, "Stability analysis of a fractional order model for the HIV/AIDS epidemic in a patchy environment," Journal of Computational and Applied Mathematics, vol. 346, pp. 323-339, 2019.

[18] M. A. Khan and A. Atangana, "Modeling the dynamics of novel coronavirus (2019-nCov) with fractional derivative," Alexandria Engineering Journal, vol. 59, no. 4, pp. 2379-2389, 2020.

[19] P. A. Naik, J. Zu, and K. M. Owolabi, "Modeling the mechanics of viral kinetics under immune control during primary infection of HIV-1 with treatment in fractional order," Physica A: Statistical Mechanics and Its Applications, vol. 545, p. 123816, 2020. 
[20] H. M. Yang, M. L. G. Macoris, K. C. Galvani, M. T. M. Andrighetti, and D. M. V. Wanderley, "Assessing the effects of temperature on dengue transmission," Epidemiology and Infection, vol. 137, no. 8, pp. 1179-1187, 2009.

[21] M. J. Keeling and P. Rohani, Modeling Infectious Diseases in Humans and Animals, Princeton University Press, Princeton, NJ, USA, 2008.

[22] C. J. Browne and S. S. Pilyugin, "Periodic multidrug therapy in a within-host virus model," Bulletin of Mathematical Biology, vol. 74, no. 3, pp. 562-589, 2012.

[23] G. P. Samanta, "Analysis of a nonautonomous HIV/AIDS epidemic model with distributed time delay," Mathematical Modelling and Analysis, vol. 15, no. 3, pp. 327-347, 2010.

[24] Y. Yang and Y. Xiao, "Threshold dynamics for an HIV model in periodic environments," Journal of Mathematical Analysis and Applications, vol. 361, no. 1, pp. 59-68, 2010.

[25] X. Liu and P. Stechlinski, "Pulse and constant control schemes for epidemic models with seasonality," Nonlinear Analysis: Real World Applications, vol. 12, no. 2, pp. 931-946, 2011.

[26] X. Wang, X. Liu, W.-c. Xie, W. Xu, and Y. Xu, "Global stability and persistence of HIV models with switching parameters and pulse control," Mathematics and Computers in Simulation, vol. 123, pp. 53-67, 2016.

[27] X. Wang, Y. Li, and W. Wang, "Stochastic stability of internal HIV models with two types of noise," Discrete Dynamics in Nature and Society, vol. 2019, Article ID 6951389, 8 pages, 2019.

[28] X. Wang, X. Liu, W. Xu, and K. Zhang, "Stochastic dynamics of HIV models with switching parameters and pulse control," Journal of the Franklin Institute, vol. 352, pp. 2756-2782, 2015.

[29] M. J. Keeling, P. Rohani, and B. T. Grenfell, "Seasonally forced disease dynamics explored as switching between attractors," Physica D: Nonlinear Phenomena, vol. 148, no. 3-4, pp. 317335, 2001.

[30] D. Liberzon and A. S. Morse, "Basic problems in stability and design of switched systems," IEEE Control Systems, vol. 19, no. 5, pp. 59-70, 1999.

[31] J. Liu, X. Liu, and W.-C. Xie, "Input-to-state stability of impulsive and switching hybrid systems with time-delay," Automatica, vol. 47, no. 5, pp. 899-908, 2011.

[32] P. A. Naik, K. M. Owolabi, M. Yavuz, and J. Zu, "Chaotic dynamics of a fractional order HIV-1 model involving AIDSrelated cancer cells," Chaos, Solitons \& Fractals, vol. 140, p. 110272, 2020.

[33] J. Danane, K. Allali, and Z. Hammouch, "Mathematical analysis of a fractional differential model of HBV infection with antibody immune response," Chaos, Solitons \& Fractals, vol. 136, p. 109787, 2020.

[34] C. A. Monje, Y. Q. Chen, B. M. Vinagre, D. Y. Xue, and V. Feliu, Fractional-Order Systems and Controls, Springer, Berlin, Germany, 2010.

[35] Z. H. Guan, D. Hill, and X. Shen, "On hybrid impulsive and switching systems and application to nonlinear control," IEEE Transactions on Automatic Control, vol. 50, pp. 1058-1062, 2005.

[36] A. Mastroberardino, Y. Cheng, A. Abdelrazec, and H. Liu, "Mathematical modeling of the HIV/AIDS epidemic in Cuba," International Journal of Biomathematics, vol. 8, no. 4, Article ID 1550047, 2015.

[37] K. M. Owolabi, "Behavioural study of symbiosis dynamics via the Caputo and AtanganaCBaleanu fractional derivatives," Chaos, Solitons and Fractals, vol. 122, pp. 89-101, 2019.

[38] C. Vargas-De-León, "Volterra-type Lyapunov functions for fractional-order epidemic systems," Communications in Nonlinear Science and Numerical Simulation, vol. 24, no. 1-3, pp. 75-85, 2015. 